\title{
The P Cygni supergiant [OMN2000] LS1 - implications for the star formation history of W51
}

\author{
J. S. Clark ${ }^{1}$, B. Davies ${ }^{2,3}$, F. Najarro ${ }^{4}$, J. MacKenty ${ }^{5}$, P. A. Crowther ${ }^{6}$, M. Messineo ${ }^{3}$, and M. A. Thompson ${ }^{7}$ \\ 1 Department of Physics and Astronomy, The Open University, Walton Hall, Milton Keynes, MK7 6AA, UK \\ 2 School of Physics and Astronomy, University of Leeds, Leeds, LS2 9JT, UK \\ 3 Chester F. Carlson Centre for Imaging Science, Rochester Institute of Technology, 54 Lomb Memorial Drive, Rochester NY 14623, \\ USA \\ 4 Departamento de Astrofísica, Centro de Astrobiología, (CSIC-INTA), Ctra. Torrejón a Ajalvir, km 4, 28850 Torrejón de Ardoz, \\ Madrid, Spain \\ 5 Space Telescope Science Institute, 3700 San Martin Drive, Baltimore, MD 21218, USA \\ 6 Department of Physics and Astronomy, University of Sheffield, Hounsfield Road, Sheffield, S3 7RH, UK \\ 7 Centre for Astrophysics Research, University of Hertfordshire, College Lane, Hatfield, AL10 9AB, UK
}

Received 3 March 2009 / Accepted 25 June 2009

\section{ABSTRACT}

\begin{abstract}
Aims. We investigate the nature of the massive star [OMN2000] LS1 and use these results to constrain the history of star formation within the host complex W51.

Methods. We utilised a combination of near-IR spectroscopy and non-LTE model atmosphere analysis to derive the physical properties of [OMN2000] LS1, and a combination of theoretical evolutionary calculations and Monte Carlo simulations to apply limits on the star formation history of W51.

Results. We find the spectrum of [OMN2000] LS1 to be consistent with that of a P Cygni supergiant. With a temperature in the range of $13.2-13.7 \mathrm{kK}$ and $\log \left(L_{*} / L_{\odot}\right) \leq 5.75$, it is significantly cooler, less luminous, and less massive than proposed by previous authors. The presence of such a star within W51 shows that star formation has been underway for at least 3 Myr, while the formation of massive $\mathrm{O}$ stars is still on going. The lack of a population of evolved red supergiants within the complex shows that the rate of formation of young massive clusters at ages $\geq 9 \mathrm{Myr}$ was lower than currently observed. We find no evidence of internally triggered, sequential star formation within W51, and favour the suggestion that star formation has proceeded at multiple indepedent sites within the GMC. Along with other examples, such as the G305 and Carina star-forming regions, we suggest that W51 is a Galactic analogue of the ubiquitous star cluster complexes seen in external galaxies such as M51 and NGC2403.
\end{abstract}

Key words. stars: evolution - ISM: H II regions - Galaxy: Open clusters and associations: general - stars: early-type

\section{Introduction}

While the physical mechanism for building massive $\left(>20 M_{\odot}\right)$ OB stars remains hotly debated, it appears likely that the majority of these stars form in star clusters (de Grijs 2005, Parker \& Goodwin 2007) rather than isolation. Moreover, observations of nearby galaxies reveal that such clusters form in larger complexes (e.g. M51; Bastian et al. 2005). Unfortunately, the process(es) that converts giant molecular clouds (GMCs) into such complexes and the timescale for their formation currently remain opaque. This in part is a consequence of the restricted spatial resolution of extragalactic studies, which compromises the determination of both stellar and cluster ages and mass functions.

Consequently, it is instructive to search for Galactic analogues of star-forming complexes that may be observed with enough resolution that individual (proto-)stars may be studied. First detected by Westerhout (1958), W51 consists of two giant $\mathrm{H}$ II regions, W51A and W51B, both of which may in turn be resolved into smaller components (e.g. Mehringer 1994; Nanda Kumar et al. 2004, and references therein). With an angular extent of $1^{\circ} \times 1^{\circ}$, and a mass of $\sim 10^{6} M_{\odot}$, W51 is amongst the most massive Galactic GMCs (Carpenter \& Sanders 1998), while the giant $\mathrm{H}$ II regions imply a large population of $\mathrm{O}$ stars to yield the requisite UV ionising flux; it therefore represents an excellent candidate for a massive star formation (SF) complex.

Near-IR imaging of W51 by Okumura et al. (2000; OMN2000) and Nanda Kumar et al. (2004) indicated a significant population of young $\mathrm{O}$ stars and massive young stellar objects (YSOs). Subsequent spectroscopic observations by Figueredo et al. (2008) and Barbosa et al. (2008) confirmed these findings, identifying several early-mid $\mathrm{O}$ stars within W51A and resolved the subregion W51 IRS2 (see Fig. 1) into a proto cluster containing an $\sim \mathrm{O} 3$ star and a massive YSO. With the recent results of Zapata et al. (2008) revealing the possible formation of a further massive $\left(\sim 40 M_{\odot}\right)$ proto star in the nearby region W51 North, it is clear that vigorous SF is currently underway within W51A.

In order to investigate the stellar content and SF history of W51 in detail we embarked on a comprehensive spectroscopic and imaging survey of the complex from near-IR to radio wavelengths. Here we present the first results of this investigation, focusing on the source [OMN2000] LS1, located at one extremity of the complex (Fig. 1; RA = $192347.64 \delta=+1436$ 38.4). OMN2000 described it as a 'P Cygni type supergiant' and 


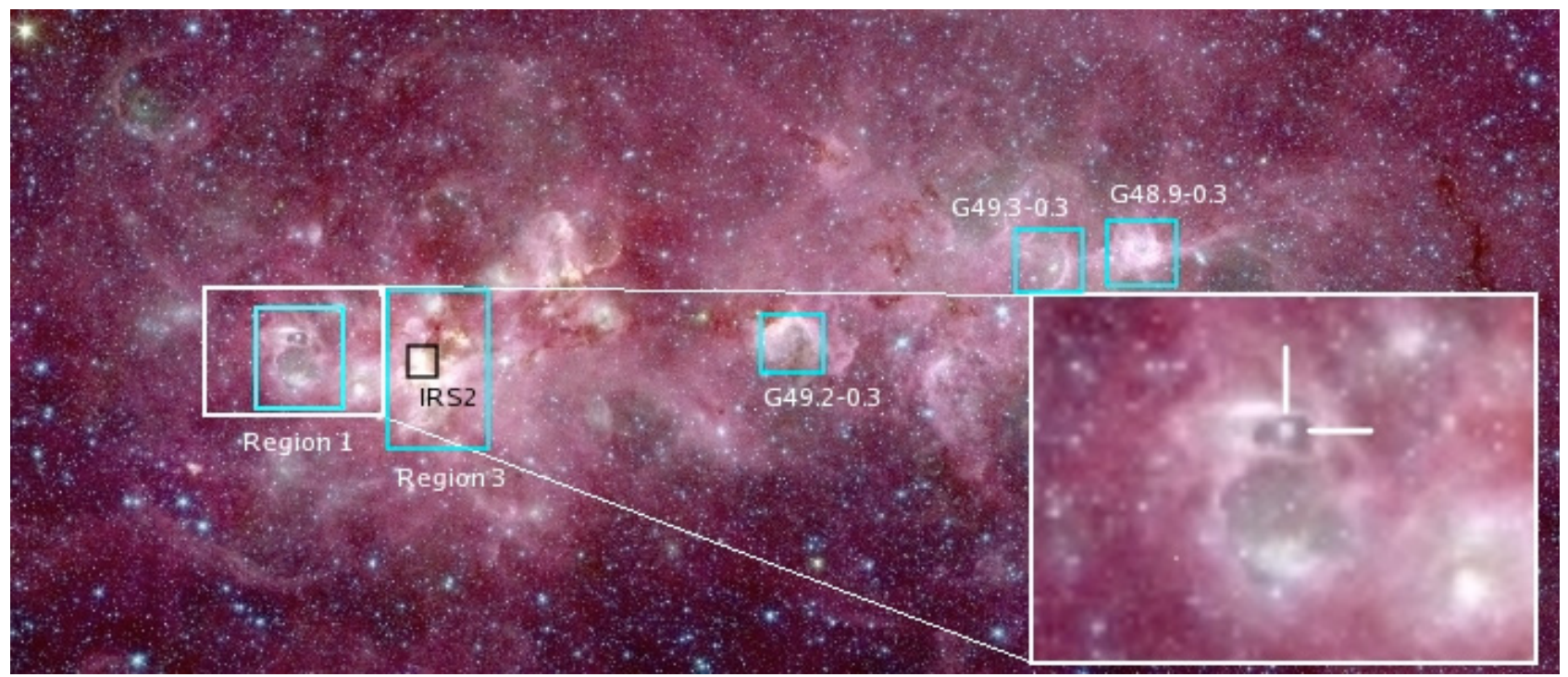

Fig. 1. Spitzer 3 colour image of W51 (blue $-3.6 \mu \mathrm{m}$, green $-4.5 \mu \mathrm{m}$ and red $-8.0 \mu \mathrm{m}$ ) with the position of [OMN2000] LS1 indicated. The overal length of the W51 complex is $\sim 100(\mathrm{~d} / 6 \mathrm{kpc}) \mathrm{pc}$, with the apparent wind blown bubble surrounding [OMN2000] LS1 being $\sim(1.8 \times 1.0)(\mathrm{d} / 6 \mathrm{kpc}) \mathrm{pc}$. For clarity we also indicate SF regions 1 and 3 as defined by OMN2000 (the latter containing the O star candidates identified by Figueredo et al. 2008), the heavily embedded massive YSO forming complex IRS2 and 3 further regions throughout the W51 complex which Nanda Kumar et al. (2004) find to host massive young stellar clusters (the fourth region studied in detail by Nanda Kumar et al. encompasses IRS 2 and is located within Region 3 of OMN2000). Note that for reasons of clarity not all the locations of star formation activity identified by these authors are indicated in this figure.

classified it as O4 I, with a progenitor mass of $\sim 120 M_{\odot}$, making it of considerable interest as one of the most massive, evolved stars in the Galaxy. In this work we present new high resolution spectroscopy of this object which allow us to better constrain its stellar properties and discuss the implications of these results for the distance to, and SF history of, W51.

\section{Data reduction and presentation}

Data was taken during the night of 27 September 2007, using the Infra-Red Multi-Object Spectrograph (IRMOS, MacKenty et al. 2003) mounted on the Mayall 4-m at the Kitt Peak National Observatory, with a 1.9 to 2.2 micron (K1) band pass filter with a grating providing $1 / \mathrm{dl}$ of 3000 . This gave us a resolution of $\sim 100 \mathrm{~km} \mathrm{~s}^{-1}$ in the the spectral range $2.0-2.2$ microns. To correct for the variability in the remaining background signal, we followed each science integration with a dark-frame of equal integration time. To compensate for variable sky background, we limited our science exposures to $2 \mathrm{~min}$. The star was dithered along the slit by 2 arcsec every five science exposures to compensate for artifacts on the detector. In total, we integrated on the object for $1 \mathrm{~h}$, with 30 individual science exposures All data reduction was done using custom-written routines in IDL. Initially, each science frame had the dark frame taken closest in time subtracted from it. The science frames were then coadded, and divided through by the normalized flat-field.

Correction for geometric distortion in both the spatial and dispersion directions are required prior to spectral extraction. To accomplish this, the data were resampled onto a linear grid. The spatial warping was characterized by fitting a 3rd degree polynomial through the spectral traces of the star in each of the dither positions. The warping in the dispersion direction was determined by linear fits to the $\mathrm{OH}$ emission lines in the sky either side of each spectral trace. Using the fits to the stellar spectral trace and the sky lines as tie-points, the warping was fitted using

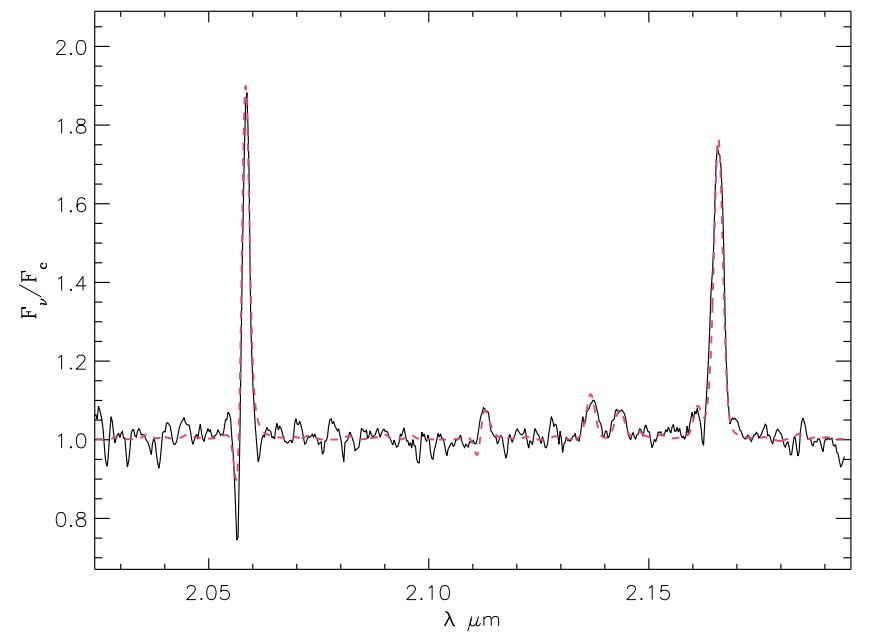

Fig. 2. High resolution $K$ band spectrum of [OMN2000] LS1 (solid line) with the best model fit superimposed (red dashed lines). The major transitions present are $\mathrm{He}$ I $2.058 \mu \mathrm{m}$ and $2.1128 \mu \mathrm{m}, \mathrm{Br} \gamma$, and $\mathrm{Mg}$ II $2.138 / 44 \mu \mathrm{m}$

a two-dimensional, third-degree polynomial. The inverse of this 2-D fit was then applied to the data to resample it onto a linear grid. As the wavelengths of the sky $\mathrm{OH}$ lines are known, the data is wavelength-calibrated in this de-warping process. The spectrum was optimally-extracted from the de-warped data using the algorithm of Horne (1986).

To remove the atmospheric absorption, the object spectrum was divided through by that of a telluric standard (SAO 107138, spectral type A8 V). Prior to division, the two spectra were first cross-correlated to correct for any sub-pixel shifts which would produce artifacts in the final spectrum. Finally, the data were normalized by the mean continuum value, and the resultant spectrum plotted in Fig. 2. 
Table 1. Derived stellar parameters for [OMN2000] LS1 as a function distance (Sects. 3.1 and 3.3).

\begin{tabular}{|c|c|c|c|c|c|c|c|c|c|c|c|c|}
\hline $\begin{array}{c}\text { Distance } \\
(\mathrm{kpc})\end{array}$ & $\begin{array}{c}R_{*} \\
\left(R_{\odot}\right)\end{array}$ & $\begin{array}{c}T_{\text {eff }} \\
(\mathrm{kK})\end{array}$ & $\log \left(L_{*} / L_{\odot}\right)$ & $\mathrm{H} / \mathrm{He}$ & $\begin{array}{c}v_{\infty} \\
\left(\mathrm{km} \mathrm{s}^{-1}\right)\end{array}$ & $\beta$ & $f(r)$ & $\begin{array}{c}\dot{M} \\
\log \left(M_{\odot} / \mathrm{yr}\right)\end{array}$ & $E(B-V)$ & $A_{K}$ & $M_{K}$ & $\begin{array}{l}\mathrm{M}_{\text {initial }} \\
\left(M_{\odot}\right)\end{array}$ \\
\hline 6 & 145.0 & 13.2 & 5.75 & $0.5 / 1.5$ & 400 & 3.0 & 0.08 & $-4.2 /-4.6$ & 3.5 & 1.2 & -8.90 & $\sim 40$ \\
\hline 3.4 & 82.5 & 13.4 & 5.30 & $0.5 / 1.5$ & 400 & 3.0 & 0.08 & $-4.6 /-5.0$ & 3.5 & 1.2 & -7.65 & $\sim 25$ \\
\hline 2 & 48.0 & 13.7 & 4.86 & $0.5 / 1.5$ & 400 & 3.0 & 0.08 & $-4.9 /-5.3$ & 3.5 & 1.2 & -6.50 & $\sim 17$ \\
\hline
\end{tabular}

As described in Sect. 3.2, $\mathrm{H} / \mathrm{He}$ ratios suffer from a modest degeneracy, resulting in a reduction in the mass loss rate by $\sim 60 \%$ for the $\mathrm{H} / \mathrm{He}=1.5$ model compared to $\mathrm{H} / \mathrm{He}=0.5$. We further estimate systematic uncertainties of $\pm 50 \mathrm{~km} \mathrm{~s}^{-1}$ in $v_{\infty}, \pm 300 \mathrm{~K}$ in $T_{\text {eff }}$ and $\pm 0.1 \mathrm{dex}$ in $\log \left(L_{*} / L_{\odot}\right)$.

\section{The nature of [OMN2000] LS1}

Comparison of the low resolution spectrum of OMN2000 to ours reveals no obvious changes in a decade. Both spectra are dominated by $\mathrm{He}$ I $2.058 \mu \mathrm{m}$ and $2.112 \mu \mathrm{m}, \mathrm{Br} \gamma$ and $\mathrm{Mg}$ II 2.138 and $2.114 \mu \mathrm{m}$ emission. However, the improved resolution and $S / N(\sim 70)$ of our observations now clearly reveals the presence of a P Cygni profile for He I $2.058 \mu \mathrm{m}$ that was hinted at in the spectrum of OMN2000; weak emission in He I 2.161 and $2.162 \mu \mathrm{m}$ likely veil a similar feature in $\mathrm{Br} \gamma$. The formal classification of O4 I by OMN2000 is incorrect, due to a lack of high excitation species such as N III and C IV (Hanson et al. 1996). However, the qualitative description as a "P Cygni supergiant" is more acccurate, with [OMN2000] LS1 bearing a close resemblance to known examples (which include confirmed and candidate Luminous Blue Variables (LBVs): Clark et al. 2003; Voors et al. 2000). While a P Cygni profile in He I $2.058 \mu \mathrm{m}$ is not seen in all such stars, it is present in HD 316285 (Hillier et al. 1998) and the Galactic Centre Ofpe/WN9 stars (Najarro et al. 1997; also known as WN9-11 h stars) which have spectra similar to known LBVs such as AG Car in the hot state. We therefore conclude that the spectrum of [OMN2000] LS1 is consistent with a a qualitative classification as a P Cygni-type B supergiant (and hence potentially a LBV).

\subsection{The distance to [OMN2000] LS1}

To derive the stellar parameters for [OMN2000] LS1 one must adopt a distance to it and, by extension, the W51 complex. Figueruedo et al. (2008) provides a summary of the results of previous studies. These show that the kinematic estimate of the distance - utilising radio recombination lines - of $5.5 \mathrm{kpc}$ (Russeil 2003), is broadly comparable to the results derived from maser proper motion measurements; $6.1 \pm 1.3 \mathrm{kpc}$ (Imai et al. 2002 ) to $8.5 \pm 2.5 \mathrm{kpc}$ (Schneps et al. 1981). However, utilising $4 \mathrm{O}$ stars which they classify as (Zero Age) Main Sequence objects, Figueruedo et al. (2008) report a significantly smaller distance to W51 of $2.0 \pm 0.3 \mathrm{kpc}$. Subsequnetly, Barbosa et al. (2008) suggest an upper limit of $5.8 \mathrm{kpc}$ by equating the radio luminosity of IRS2 with the ionising flux from the $\mathrm{O}$ star W51d under the assumption that it too is a Main Sequence star. Finally and most recently, $\mathrm{Xu}$ et al. (2008) report a distance of $5.1_{-1.4}^{+2.9} \mathrm{kpc}$ based on trigonometric parallax measurements.

Both Figueredo et al. (2008) and Xu et al. (2008) recognise the difficulty in reconciling the spectroscopic distance with the other estimates but are unable to provide an explanation for this discrepancy. Possible reasons for underestimating the spctroscopic distance would be the adoption of an incorrect reddening law to W51, unrecognised binarity or multiplicity in the stars or an incorrect spectral classification. Relating to the final point we note that the luminosity of early $\mathrm{O}$ stars may be determined from the Br $\gamma$ line (Hanson et al. 2005), with supergiants demonstrating infilling or emission. While the emission observed in three of the four stars studied by Figueredo et al. (2008) may result from incomplete nebular subtraction, if the stars were Main Sequence objects, the stark absorption wings in the $\operatorname{Br} \gamma$ profile would be visible given the $S / N$ and resolution of the spectra. Thus we suggest that the luminosity class of these stars - adopted due to their proximity to a star forming region and hence their distance may be underestimated.

For a distance to W51 of $6 \mathrm{kpc}$, the $\mathrm{O}$ stars discussed by Figueredo et al. (2008) are at projected distances of 2-4 pc from the compact star forming region IRS2. While it might appear unlikely for evolved stars to be located so close to regions of ongoing SF, early O supergiants are found within the massive star forming regions G305 (Leistra et al. 2005) and W43 (Blum et al. 1999). Thus non-coevality of the stellar population(s) may be a common feature of large star forming complexes such as W51 and G305 (Davies et al., in prep.). Nevertheless, given this uncertainty, we undertook our analysis of [OMN2000] LS1 for both near, spectroscopic $(2 \mathrm{kpc})$ and far, kinematic and parallactic distances (for which we adopted a representative value of $6 \mathrm{kpc})$ as well as for a third, intermediate $(3.4 \mathrm{kpc})$ distance, the choice of which is justified in Sect. 3.3.

\subsection{Physical parameters of [OMN2000] LS1}

We have used CMFGEN, the iterative, non-LTE line blanketing method presented by Hillier \& Miller (1998) to model [OMN2000] LS1 and estimate the physical properties of the star. The method solves the radiative transfer equation in the co-moving frame and in spherical geometry for the expanding atmospheres of early-type stars. The model is prescribed by the stellar radius, $R_{*}$, the stellar luminosity, $L_{*}$, the mass-loss rate $\dot{M}$, the velocity field, $v(r)$ (defined by $v_{\infty}$ and $\beta$ ), the volume filling factor characterizing the clumping of the stellar wind, $f(r)$, and elemental abundances. Hillier \& Miller $(1998,1999)$ present a detailed discussion of the code.

For the present analysis, we have assumed solar metalicities for an atmosphere composed of $\mathrm{H}, \mathrm{He}, \mathrm{C}, \mathrm{N}, \mathrm{O}, \mathrm{Mg}, \mathrm{Si}, \mathrm{S}, \mathrm{Fe}$ and Ni. Observational constraints are provided by the $K$-band spectrum and the 2MASS $J, H$ and $K$ photometry. The validity of our technique has been demonstrated in Najarro et al. (1999) and Najarro (2001) by calibrating our method against stars with similar spectral type such as P Cygni and HDE 316285 (for which optical and UV spectra are also available). We refer to Najarro et al. (2008) for a detailed review on the analysis technique and present the results from fits for the three distances adopted in Table 1. As described by Najarro et al. (2008), the computationally intensive analysis precludes sufficient numbers of models being calculated to permit statistically robust error estimates to be made for all parameters. Consequently, the uncertainties presented in Table 1 represent the range of values for which an acceptable fit to the available data is acheived, an approach also adopted by Martins et al. (2007). 
The primary diagnostics used were the presence and relative strengths of the three He I emission lines at $2.058 \mu \mathrm{m}, 2.112 \mu \mathrm{m}$, $2.185 \mu \mathrm{m}$, the He I (7-4) complex around $2.16 \mu \mathrm{m}, \mathrm{Br} \gamma$ and the $\mathrm{Mg}$ II lines $(2.138 \mu \mathrm{m}$ and $2.144 \mu \mathrm{m})$. A lower temperature solution may be excluded due to the lack of any Fe II semi-forbidden lines (seen in emission for cooler P Cygni supergiants; Geballe et al. 2000), while the He I $2.058 \mu \mathrm{m}$ line would also be expected to be significantly weaker. Conversely, higher temperatures would lead to significantly stronger emission in this line - both in absolute terms and also in relation to $\operatorname{Br} \gamma$ - than is observed.

P Cygni supergiants with high mass loss rates potentially demonstrate a degeneracy between the $\mathrm{H} / \mathrm{He}$ ratio and mass loss rate. This occurs when the combination of stellar temperature and high wind density causes He II to recombine to He I very close to the photosphere. In such a situation any $\mathrm{He} / \mathrm{H}$ ratio may fit the observations with an appropiate scaling of the mass loss rate and a small variation of the stellar temperature; this effect is observed in e.g. HD 316285 (see Hillier et al. 1998, for a discussion). However, unlike this star, the lower mass loss rate for [OMN2000] LS1 leads to a reduced wind density which, when combined with a higher effective temperature, minimises the effect of this degeneracy. $\mathrm{H} / \mathrm{He}$ ratios of 0.5 to 1.5 are permitted by our modeling; if $\mathrm{H} / \mathrm{He}$ were higher then the $\mathrm{He}$ I 2.112 and $2.185 \mu \mathrm{m}$ lines and He I (7-4) complex would be weaker than observed. Following the scaling determined by Hillier et al. (1998) for HD 316285, these abundances correspond to a reduction in the mass loss rate of $\sim 60 \%$ for $\mathrm{H} / \mathrm{He}=1.5$ when compared to that determined for $\mathrm{H} / \mathrm{He}=0.5$ (Table 1 ) but only a small change $(<300 \mathrm{~K})$ in temperature. For this parameter regime, $\tau \sim 2 / 3$ is already reached at the base of the wind, and so no information is obtained from the hydrostatic layers of the star, the strong wind consequently fully determining $T_{\text {eff }}$ (which is defined at $\tau \sim 2 / 3$ ).

The value of the reddening presented in Table 1 was determined via comparison of the predicted stellar near IR colour to 2MASS photometry of the source in order to determine the near IR excess due to reddening, $E(J-K)$. A value of $A_{K}$ was then calculated via the relation $A_{K}=0.67 E(J-K)$ and finally converted to $E(B-V)$ via the relations $0.112 A_{V}=A_{K}$ and $A_{V}=3.1 E(B-V)$ (Rieke \& Lebofsky 1985). The random error for the observed $(J-K)$ from the 2 MASS data is small $( \pm 0.03 \mathrm{mag})$, as is the systematic uncertainty in the intrinsic $(J-K)_{\mathrm{o}}$ colour of the star (due to the range of mass loss rates permitted by the modeling); we conservatively estimate an uncertainty in $A_{K}$ of $\pm 0.1 \mathrm{mag}$. The well developed P Cygni profile in He I $2.058 \mu \mathrm{m}$ permits a determination of the terminal velocity of the wind $\left(v_{\infty}\right)$ of $\pm 50 \mathrm{~km} \mathrm{~s}^{-1}$. Finally, we assume an uncertainty in $\log \left(L_{\text {bol }}\right)$ of \pm 0.1 dex, which we regard as highly conservative, given the small systematic uncertaintites in both reddening and the temperature dependant bolometric correction.

\subsection{The evolutionary state of [OMN2000] LS1}

Based on the near-IR magnitudes of [OMN2000 LS1] and the properties of the wind blown bubble surrounding it ( $\mathrm{H}$ II region j), OMN2000 propose $T_{\text {eff }} \sim 40 \mathrm{kK}$ and $\log \left(L_{*} / L_{\odot}\right) \sim 6.3$, yielding an initial mass of $\sim 120 M_{\odot}$. Irrespective of whether a distance of 2 or $6 \mathrm{kpc}$ is correct, we find these values to be significant overestimates. We may also use our results to discriminate between the two distance estimates on the grounds of self consistency from both observational and theoretical perspectives.

Firstly, the $K$ band spectrum suggests a classification as a $\mathrm{P}$ Cygni supergiant. If it were located at $2 \mathrm{kpc}$, [OMN2000 LS1] would have a luminosity $\geq 0.4$ dex below the lower end of the range observed for such stars $\left(\log \left(L_{*} / L_{\odot}\right) \sim 5.3\right.$ as seen for HD 168625; Clark et al. 2005b). In contrast the resultant luminosity at $6 \mathrm{kpc}$ is entirely consistent with such a spectral classificaton. Moreover the other physical parameters such as mass loss rate, terminal wind velocity and $\mathrm{H} / \mathrm{He}$ ratio are also fully consistent with those derived from non-LTE modeling of other P Cygni-type supergiants such as P Cygni, HD 316285 (Hillier et al. 1998) and AG Car (Groh et al. 2009) and the closely related WN9-11 stars (e.g. Najarro et al. 1997; Martins et al. 2007).

With the temperatures and luminosities given in Table 1, comparison to the evolutionary tracks of Meynet \& Maeder (2000) suggest initial masses in the region of $\sim 40 M_{\odot}$ and $\sim 17 M_{\odot}$ for $6 \mathrm{kpc}$ and $2 \mathrm{kpc}$ respectively (Table 1 ). While it is thought that stars between $25-40 M_{\odot}$ will encounter a P Cygni supergiant phase during their post-red supergiant (RSG) bluewards evolution across the HR diagram, it is not expected that lower mass stars will evolve in such a manner, instead exploding as SNe while RSGs, again favouring a larger distance.

We may test this theoretical prediction via observations of the stellar populations of young, massive coeval clusters. Several P Cygni supergiants have been identified in Westerlund 1 ( 4-5 Myr; Clark et al. 2005) and the Galactic Centre ( $6 \mathrm{Myr}$; Najarro et al. 1997; Martins et al. 2007; Paumard et al. 2006), Quintuplet ( $\sim 4 \pm 1$ Myr; Figer et al. 1999) and 1806-20 ( 3-5 Myr; Figer et al. 2005; Bibby et al. 2008) clusters. In all cases the stellar contents and ages of these clusters are consistent with progenitor masses for these stars in the $\sim 30-60 M_{\odot}$ range. In contrast, despite a sample size of 30 clusters which are massive enough to host blue and/or red supergiants and have ages in excess of 10 Myr (Eggenberger et al. 2002; Davies et al. 2007, 2008) - appropiate for the post-Main Sequence (MS) evolution of stars of $\leq 20 M_{\odot}$ - to the best of our knowledge to date no P Cygni supergiant has been identified within any of them ${ }^{1}$. This is even true for such massive, well stocked clusters as RSGC1 and 2 (Davies et al. 2008) and we therefore conclude that stars of such relatively low masses do not pass through such a phase at solar metalicities.

Thus, while we cannot a priori exclude the possibility that the P Cygni supergiant phase occurs at sufficiently low $\left(\leq 20 M_{\odot}\right)$ masses to be reconciled with a distance to [OMN2000 LS1] of $2 \mathrm{kpc}$, we suggest that a distance of $\sim 6 \mathrm{kpc}$ is more consistent with current observational and theoretical constraints on the nature of such stars and the majority of current distance estimates for W51, including the recent parallactic estimate of $\mathrm{Xu}$ et al. (2008). Finally, requiring the luminosity of [OMN2000 LS1] to match that of the faintest P Cygni supergiant identified to date $\left(\log \left(L_{*} / L_{\odot}\right) \sim 5.3\right.$; see above) results in a distance of $3.4 \mathrm{kpc}$ (Table 1). Such a luminosity would correspond to an initial stellar mass of $\sim 25 M_{\odot}-$ a value on the cusp of that required by theoretical predictions for a star to evolve bluewards after exiting the RSG phase and hence potentially become a P Cygni supergiant $^{2}$.

We conclude that [OMN2000] LS1 is an evolved massive star in a P Cygni supergiant phase, with a high mass loss rate and evidence for chemical enrichment. While such a classification

\footnotetext{
1 Note that the intrinsic luminosity and strong emission line spectrum of a P Cygni supergiant would identify them in either a spectroscopic or a broad+narrow (e.g. $\mathrm{H} \alpha$ ) band imaging survey.

2 Indeed two clusters in the Large Magellanic Cloud are found to contain the closely related Ofpe/WN9 stars; LH39 ( 8-10 Myr) and LH89 ( 5-7 Myr); both ages from Massey et al. (2000). Thus it appears that under certain conditions stars with masses as low as $\sim 25 M_{\odot}$ may become P Cygni supergiants.
} 
does not premit an exact distance to be determined, we favour a distance compatable with recent kinematic and parallactic estimates to W51, which would imply a progenitor mass of the order of $\sim 40 M_{\odot}$.

\section{The star formation history of W51}

Combined with the identification of massive protostars, the presence of [OMN2000] LS1 within W51A indicates that massive star formation has been underway for a significant period of time. OMN2000 report ages of only 1.9 Myr and 2.3 Myr for [OM2000] LS1 and the region of W51 which hosts it (their "Region 1"; Fig. 1). However, comparison of our modelling results to the theoretical predictions of Meynet \& Maeder (2000) suggest this is an underestimate, with the episode of SF that yielded [OMN2000] LS1 occuring at least 3 Myr ago. Precise limits depend on both distance and stellar rotation, which is currently unquantifiable, but both theoretical and observational considerations suggest 3-6 Myr for a distance of $6 \mathrm{kpc}$ and $>10 \mathrm{Myr}$ for $2 \mathrm{kpc}$, if such a low luminosity solution is tenable (with the intermediate value of $3.4 \mathrm{kpc}$ implying a range of $\sim 5-10 \mathrm{Myr}$ ).

In an analagous manner, the numbers and ratios of $\mathrm{OB}$ supergiants to Wolf Rayets and cool evolved stars such as Yellow Hypergiants and RSGs also potentially constrain recent ( 20 Myr) SF within W51. Hadfield et al. (2007) showed that hot post-MS evolutionary phases may be identified by an IR excess due to stellar winds. While a number of stars with such excesses are found within the W51 complex (van Dyk et al. 2008, priv. comm.), photometric data alone do not allow for the discrimination of their precise evolutionary state, which is necessary to constrain the SF history ${ }^{3}$. However, cool evolved stars such as RSGs are readily identifiable even at a distance of $6 \mathrm{kpc}$ and $\mathrm{A}_{v}=25 \mathrm{mag}$ (Davies et al. 2008). Examination of the 2MASS data for the complex reveals only a single bright candidate (2MASS J19225290+1411210; $J=7.21, H=5.50$, $K=4.63)$, with colours consistent with a moderately reddened M star $\left(A_{v} \sim 8\right.$; colours from Elias et al. 1985), for which we infer $\log \left(L / L_{\odot}\right) \sim 5.5$ (5.0) for a putative spectral type of M0 I (M5 I; bolometric corrections from Levesque et al. 2005) at a distance of $6 \mathrm{kpc}$ and hence an age in the range of 10-14 Myr (e.g. Davies et al. 2008), noting that the comparatively low extinction could be the result of clearing of the local ISM by precursor $\mathrm{O}$ stars (as suggested by the anonymous referee).

Irrespective of the actual classification of 2MASS J19225290+1411210, a large population of RSGs appears to be absent from W51. Following the methodology of Davies et al. (2008, and refs. therein) we may utilise this observation to interpret the prior SF history. We built synthetic clusters of differing masses in the $1-40000 M_{\odot}$ range, populating them according to a Kroupa type Initial Mass Function (Kroupa 2001) ${ }^{4}$, and evolving stars according to the rotating $\left(v_{\text {rot }}=300 \mathrm{~km} \mathrm{~s}^{-1}\right)$, solar metalicity evolutionary tracks of Meynet \& Maeder (2000). RSGs were classified as those stars with $\log \left(L / L_{\odot}\right)>4.5$ and $T<4500 \mathrm{~K}$, and 200 trials per age interval were employed to reduce random noise. Results from these simulations, in terms

\footnotetext{
3 There exists the potential for the miss-identification of pre-MS objects as post-MS stars, with Hadfield et al. (2007) finding a large number of $\mathrm{B}[\mathrm{e}]$ stars using their IR selection criteria; a heterogeneous classification containing stars at very different evolutionary stages (Lamers et al. 1998).

4 Previous simulations of RSGC1-3 (Davies et al. 2007, 2008; Clark et al. 2009) had assumed a Salpeter IMF - for a given population of RSGs the Kroupa IMF yeilds more low mass stars, resulting in an increase in cluster mass by $\sim 30 \%$ over the estimates given in these works.
}

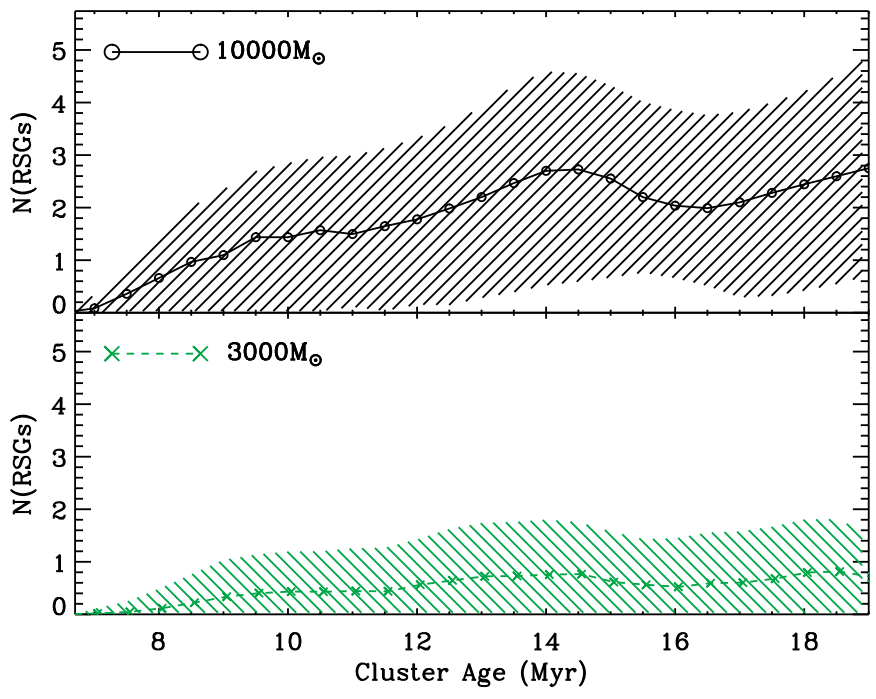

Fig. 3. Plot showing the mean number of RSGs expected for clusters with masses of 3 and $10 \times 10^{3} M_{\odot}$ as a function of cluster age, the hashed regions indicating the formal $1 \sigma$ range derived from the Monte Carlo simulations. By comparison, current SF within W51 is forming clusters in the $2-10 \times 10^{3} M_{\odot}$ range, and the Orion Nebula cluster is $\sim 2000 M_{\odot}$ (Sect. 4). The dip between 14-20 Myr is caused by the onset of a blue loop for a restricted mass range of progenitors.

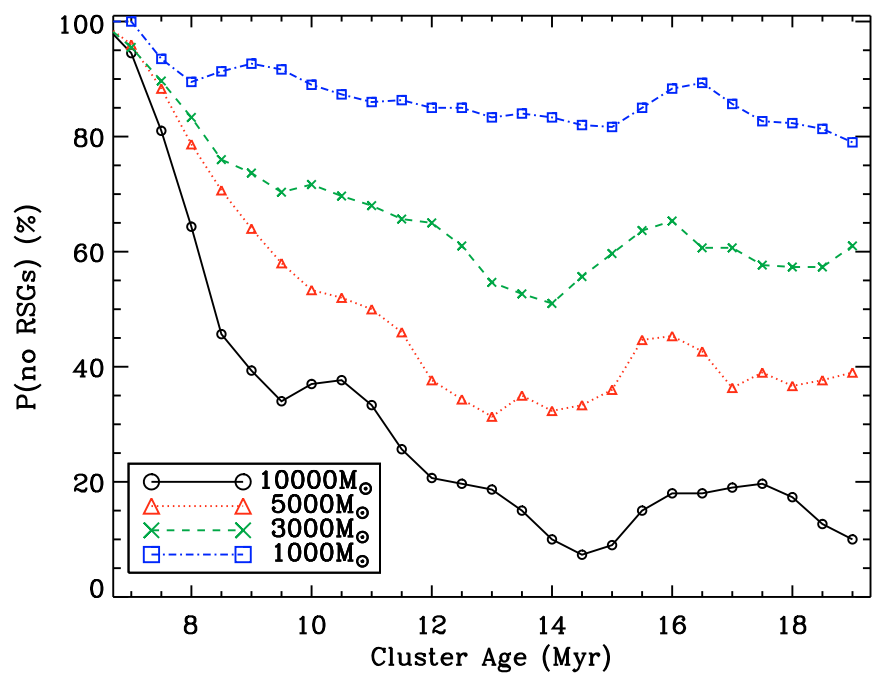

Fig. 4. Plot showing the probability that a cluster of given age and mass has no RSGs present (Sect. 4).

of both the mean number of RSGs expected and the probability that none will be present, as a function of cluster mass and age, are presented in Figs. 3 and 4.

These results clearly demonstrate that, given the brevity of the RSG phase, statistically significant conclusions may only been drawn for very massive $\left(\gg 10^{4} M_{\odot}\right)$ clusters which, by extention, may host correspondingly rich populations of RSGs. Indeed, RSGs are so rare at ages of $<8$ Myr that no meaningful constraints may be placed on the properties of individual host clusters, even for such extreme masses. These uncertainties are further exacerbated in the event that constraints on the age of the RSG population are unavailable, which would occur if no estimate of the stellar luminosity were available. Since this would be the case for 2MASS J19225290+1411210 if it were a $R S G$, we may only infer that any putative natal cluster must be greater than 5 Myr in age. However, we may compare the results of 
the Monte Carlo simulations to the properties of the ensemble of clusters identified by Nanda Kumar et al. (2004) to investigate whether the global rate of SF in the W51 complex was larger or smaller in the past. Nanda Kumar et al. (2004; Fig. 1) reported the discovery of 5 massive $\left(2-10 \times 10^{3} M_{\odot}\right)$, young (0.7-3 Myr) clusters in the 4 fields they surveyed. Given the results presented in Fig. 4, if these clusters were to be observed at an age of 9(20) Myr we would expect a total of 3(20) RSGs to be present, with only a $<5 \%$ chance that none would be present at either epoch. We therefore conclude that the current SF differs from past ( $>9 \mathrm{Myr}$ ) epochs in the sense that greater numbers of massive clusters are currently being formed.

While we defer a detailed description of the Spitzer data to a future paper, we note that the morphology of the emission also casts doubt on the hypothesis of OMN2000 that SF in their Region 1 subsequently triggered similar activity in their Region 3 (which contains the massive proto-cluster IRS2; Figueredo et al. 2008; Barbosa et al. 2008). Under such a scenario we might expect to see a mid-IR morphology similar to that of the G305 SF complex (Clark \& Porter 2004), with IRS2 residing on the periphery of a large wind blown cavity, which would be readily visible in e.g. Spitzer data (Churchwell et al. 2006). While such structures are found within Region 1 of OMN2000 (Fig. 1), IRS2 clearly resides beyond their boundaries, implying that it formed independently of this activity.

Therefore, in summary we are able to conclude that SF activity within the W51 complex resulting in the production of massive $\mathrm{O}$ stars is ongoing and has proceeded at multiple sites throughout the cloud over at least the last $\sim 3 \mathrm{Myr}$, with no current evidence for widespread internal sequential triggering, although the simultaneity of these events does suggest a large scale external trigger (c.f Nanda Kumar et al. 2004).

Recent observations of external galaxies such as M51 have revealed that SF appears to yield complexes of star clusters with a range of ages ( 10 Myr; Bastian et al. 2005) and it appears likely that W51, along with other complexes such as G305 (Clark \& Porter 2004) and the Carina Nebula (Smith \& Brooks 2007) are Galactic analogues. However, despite the physical similarities demonstrated by these complexes - a heirarchical distribution of star formation on multiple spatial scales which likely reflect the fractal nature of their natal GMCs (Elmegreen 2008) - differences between the locations of distinct stellar populations are apparent. For example the extragalactic Giant HII region NGC2403-I consists of a halo of 7-10 Myr old RSGs surrounding a population of significantly younger (2-6 Myr) massive stellar clusters (Drissen et al. 1999). Conversely 30 Dor and the G305 complex comprise dense central clusters (2-5 Myr) which are triggering new waves of SF $(\leq 1 \mathrm{Myr})$ on the periphery of wind blown bubbles (Walborn et al. 2002; Clark \& Porter 2004), while we currently find no evidence for spatially segregated, sequentially triggered star formation in W51.

\section{Conclusions}

Utilising new near-IR spectroscopic observations we find that [OMN2000] LS1 is a massive evolved star best classified as an extreme P Cygni B supergiant. Our non-LTE analysis of the star suggests a significant downwards revision in stellar temperature, luminosity and initial mass when compared to the values presented by OMN2000. Assuming the spectroscopically determined distance of $2 \mathrm{kpc}$ to W51 (Figueredo et al. 2008) results in a luminosity and progenitor mass significantly lower than expected for such stars on both observational and theoretical grounds. In contrast, a distance of $6 \mathrm{kpc}-$ representative of both kinematic and parallactic estimates - results in a luminosity of $\log \left(L / L_{\odot}\right) \sim 5.75$, an initial mass of order $\sim 40 M_{\odot}$, a mass loss rate of $6.6 \times 10^{-5} M_{\odot} \mathrm{yr}^{-1}$ and and elevated $\mathrm{He} / \mathrm{H}$ ratio; entirely consistent with quantitative analyses of other P Cygni supergiants, LBVs and the closely related WN9-11 stars. Given a current empirical minimum luminosity of $\log \left(L / L_{\odot}\right) \sim 5.3$ for the P Cygni supergiants, we suggest a corresponding minimum distance of $\sim 3.4 \mathrm{kpc}$ for [OMN2000] LS1.

Nevertheless, for either distance, this result demonstrates that massive SF in W51 has been underway for a minimum of $3 \mathrm{Myr}$ and is still ongoing (e.g. Figueredo et al. 2008). However, the lack of a significant population of RSGs - if indeed any are present - within the complex suggests that the formation of star clusters differed in the past, such that the massive clusters being formed now (Nanda Kumar et al. 2004) were not forming $\geq 9$ Myr ago.

The morphology of the mid-IR emission surrouding [OMN2000] LS1 casts doubt upon the hypothesis of OMN2000 that sequential, internally triggered SF has occurred within W51, but is consistent with the suggestion of Nanda Kumar et al. (2004) of activity at multiple sites initiated by an external trigger. In this respect W51 differs from other complexes such as G305 and the Carina nebula, where ongoing SF triggered by the first generation of stars appears to be occuring on the periphery of a wind blown cavity in the GMC.

Nevertheless, all three regions appear to be Galactic counterparts to the star forming complexes identified in external galaxeis such as M51 (Bastian et al. 2005) and NGC2403 (Drissen et al. 1999), which are characterised by SF on multiple spatial scales and over a comparatively short $(\sim 10 \mathrm{Myr})$ period of time. While the global, heirarchical properties of such complexes likely reflect the initial conditions of the natal GMCs (Elmegreen 2008), the relative magnitudes of, and interplay between, internal (feedback from OB stars and SNe) and external triggering processes ( $\mathrm{SNe}$, passage of galactic density waves and interaction with external galaxies) presumably play a key role in determining the detailed SF history and morphology of individual examples.

Acknowledgements. J.S.C. is funded by an RCUK fellowship. F. Najarro acknowledges Spanish grants AYA2008-06166-C03-02 and GTC Consolider. IRMOS was developed with the generous support of the Space Telescope Science Institute, the James Webb Space Telescope, the NASA Goddard Space Flight Center, the Kitt Peak National Observatory. The National Optical Astronomy Observatory (NOAO) consists of Kitt Peak National Observatory near Tucson, Arizona, Cerro Tololo Inter-American Observatory near La Serena, Chile, and the NOAO Gemini Science Center. NOAO is operated by the Association of Universities for Research in Astronomy (AURA) under a cooperative agreement with the National Science Foundation.

\section{References}

Barbosa, C. L., Blum, R. D., Conti, P. S., Damineli, A., \& Figueredo, E. 2008, ApJ, 678, L55

Bastian, N., Gieles, M., Efremov, Y. N., \& Lamers, H. J. G. L. M. 2005, A\&A, 443, 79

Bibby, J., Crowther, P. A., Furness, J., \& Clark, J. S. 2008, MNRAS, 386, L23 Blum, R. D., Damineli, A., \& Conti, P. S. 2000, AJ, 117, 1392

Carpenter, J. M., \& Sanders, D. B. 1998, AJ, 116, 1856

Clark, J. S., \& Porter, J. M. 2004, A\&A, 427, 839

Clark, J. S., Egan, M. P., Crowther, P. A., et al. 2003, A\&A, 412, 185

Clark, J. S., Negueruela, I., Crowther, P. A., \& Goodwin, S. P. 2005a, A\&A, 434, 949

Clark, J. S., Larionov, V. M., \& Arkharov, A. 2005b, 435, 239

Clark, J. S., Negueruela, I., Davies, B., et al. 2009, A\&A, 498, 109

Churchwell, E. B., Povich, M. S., \& Allen, D. 2006, ApJ, 49, 759

Davies B., Figer, D. F., Kudritzki, R.-P., et al. 2007, ApJ, 671,781

Davies B., Figer, D. F., Law, C. J., et al. 2008, ApJ, 676, 1016 
de Grijs, R., 2005, in Proc. The Formation and Evolution of Massive Young Star Clusters, ed. H. J. G. L. M. Lamers, L. J., Smith \& A. Nota, ASP Conf. Ser., 322,29

Drissen L., Roy, J.-R., Moffat, A. F. J., \& Shara, M. M. 1999, AJ, 117, 1249

Eggenberger, P., Meynet, G., \& Maeder, A. 2002, A\&A, 386, 576

Elias, J. H., Frogel, J. A., \& Humphreys, R. M. 1985, ApJS, 57, 91

Elmegreen, B. G. 2008, ApJ, 672, 1006

Figer, D. F., Morris, M., Geballe, T. R., et al. 1999, ApJ, 525, 759

Figer, D. F., Najarro, F., Geballe, T. R., Blum, R. D., \& Kudritzki, R. P. 2005, ApJL, 622, L49

Figueredo, E., Blum, R. D., Damineli, A., Conti, P. S., \& Barbosa, C. L. 2008, AJ, 136, 221

Geballe, T. R., Najarro, F., \& Figer, D. F. 2000, ApJL, 530, L97

Groh, J. H., Hillier, D. J., Damineli, A., et al. 2009, ApJ, 698, 1698

Hadfield, L. J., van Dyk, S. D., Morris, P. W., et al. 2007, MNRAS, 376, 248

Hanson, M. M., Conti, P. S., \& Rieke, M. J. 1996, ApJS, 107, 281

Hanson, M. M, Kudritzki, R.-P., Kenworthy, M. A., Puls, J., \& Tokunaga, A. T. 2005, ApJS, 161, 154

Hillier, D. J., \& Miller, D. L. 1998, ApJ, 496, 407

Hillier, D. J., \& Miller, D. L. 1999, ApJ, 519, 354

Hillier, D. J., Crowther, P. A., Najarro, F., \& Fullerton, A. W. 1998, A\&A, 340, 483

Horne, K. 1986, PASP, 98, 609

Kroupa, P. 2001, MNRAS, 322, 231

Lamers, H. J. G. L. M., Zickgraf, F.-J., de Winter, S., Houziaux, L., \& Zorec, J. 1998, A\&A, 340, 117

Imai, H., Watanabe, T., \& Omodaka, T., 2002, PASJ, 54, 741

Leistra, A., Cotera, A. S., Leibert, J., \& Burton, M. 2005, AJ, 130, 1719
Levesque, E. M., Massey, P., Olsen, K. A. G., et al. 2005, ApJ, 628, 973

MacKenty, J. W., Greenhouse, M. A., Green, R. F., et al. 2003, proc SPIE, 4841, 953

Martins, F., Genzel, R., Hillier, D. J., et al. 2007, ApJ, 468, 233

Massey, P., Waterhouse, E., \& DeGioia-Eastwood, K. 2000, AJ, 119, 2214

Mehringer, D. M. 1994, ApJS, 91, 713

Meynet, G., \& Maeder, A. 2000, A\&A, 361, 101

Najarro, F. 2001, P Cygni 2000: 400 Years of Progress, ASPC, 233, 133

Najarro, F., Krabbe, A., Genzel, R., et al. 1997, A\&A, 325, 700

Najarro, F., Hillier, D. J., Figer, D. F., \& Geballe, T. R. 1999, The Central Parsecs of the Galaxy, ASPC, 186, 340

Najarro, F., Figer, D. F., Hillier, D. J., Geballe, T.R., \& Kudritzki, R. P. 2009, ApJ, 691, 1816

Nanda Kumar, M. S., Kamath, U. S., \& Davis, C. J. 2004, MNRAS, 353, 1025

Okumura, S., Mori, A., Nishihara, E., Watanabe, E., \& Yamashita, T. 2000, ApJ, 543, 799 (OMN2000)

Parker, R. J., \& Goodwin, S. P. 2007, MNRAS, 380, 1271

Paumard, T., Genzel, R., \& Martins, F., et al. 2006, ApJ, 643, 1011

Rieke, G. H., \& Lebofsky, M. J. 1985, ApJ, 288, 618

Russeil, D. 2003, A\&A, 397, 133

Schneps, A., Lane, A. P., Downes, D., et al., 1981, ApJ, 249, 124

Smith, N., \& Brooks, K. J. 2007, MNRAS, 3798, 1279

Voors, R. H. M., Waters, L. B. F. M., de Koter, A., et al. 2000, A\&A, 356, 501

Walborn, N. R., Maiz-Apellaniz, J., \& Barba, R. H. 2002, AJ, 124, 1601

Westerhout, G., 1958, Bulletin of the Astronomical Institute of the Netherlands, $14,215$.

Xu, Y., Reid, J., Menten, K. M., et al. 2008, ApJ, in press

Zapata, L. A., Palau, A., \& Ho, P. T. P. 2008, A\&A, 479, L25 